\title{
ON THE STABILITY OF MINIMAL CONES IN WARPED PRODUCTS
}

\author{
K. S. BEZERRA, A. CAMINHA, AND B. P. LIMA
}

\begin{abstract}
In a seminal paper published in 1968, J. Simons proved that, for $n \leq 5$, the Euclidean (minimal) cone $C M$, built on a closed, oriented, minimal and non totally geodesic hypersurface $M^{n}$ of $\mathbb{S}^{n+1}$ is unstable. In this paper, we extend Simons' analysis to warped (minimal) cones built over a closed, oriented, minimal hypersurface of a leaf of suitable warped product spaces. Then, we apply our general results to the particular case of the warped product model of the Euclidean sphere, and establish the unstability of $C M$, whenever $2 \leq n \leq 14$ and $M^{n}$ is a closed, oriented, minimal and non totally geodesic hypersurface of $\mathbb{S}^{n+1}$.
\end{abstract}

\section{INTRODUCTION}

In 1968, J. Simons (cf. [10]) generalized a theorem of F. J. Almgren, Jr. (cf. [1]), showing that, for $n \leq 5$, the Euclidean cone built over any closed, minimal and non totally geodesic hypersurface of $\mathbb{S}^{n+1}$ is a minimal unstable hypersurface of $\mathbb{R}^{n+2}$.

If $M^{n}$ is a hypersurface of $\mathbb{S}^{n+1}$, the Euclidean cone $C M$ over $M$ is given by the immersion $\Phi: M^{n} \times(0,1] \rightarrow \mathbb{R}^{n+2}$, that sends $(p, t)$ to $t p$. For $0<\epsilon<1$, the $\epsilon$ truncated cone $C_{\epsilon} M$ over $M$ is the restriction of $\Phi$ to $M \times[\epsilon, 1]$. In [10, it is shown that, if $M^{n}$ is a closed minimal hypersurface of $\mathbb{S}^{n+1}$, then $C M \backslash\{0\}$ is a minimal hypersurface of $\mathbb{R}^{n+2}$; moreover, $C_{\epsilon} M$ is compact and such that $\partial\left(C_{\epsilon} M\right)=M \cup M_{\epsilon}$, where $M_{\epsilon}=\{\epsilon p ; p \in M\}$.

In 4, the second author extended this notion in the following way: let $\bar{M}_{c}^{n+2}$ be a Riemannian manifold whose sectional curvature is constant and equal to $c$. Assume that $\bar{M}$ admits a closed conformal vector field $\xi \in \mathfrak{X}(\bar{M})$, with conformal factor $\psi_{\xi}$. If $\xi \neq 0$ on $\bar{M}$, it is well known that the distribution $\{\xi\}^{\perp}$ is integrable, with leaves totally umbilical in $\bar{M}$. Let $\Xi^{n+1}$ be such a leaf and $\varphi: M^{n} \rightarrow \Xi^{n+1}$ be a closed hypersurface $\Xi$. If $\Psi$ denotes the flow of $\xi /\|\xi\|$, the compactness of $M$ guarantees the existence of $\epsilon>0$ such that $\Psi$ is well defined in $[-\epsilon, 0] \times \varphi(M)$, and the mapping $\Phi: M^{n} \times[-\epsilon, 0] \rightarrow \bar{M}^{n+2}$ that sends $(p, t)$ to $\Psi(t, \varphi(p))$ is also an immersion. By furnishing $M^{n} \times[-\epsilon, 0]$ with the induced metric, we turn $\Phi$ into an isometric immersion such that $\Phi_{\mid M^{n} \times\{0\}}=\varphi$; the Riemannian manifold $M^{n} \times[-\epsilon, 0]$, is the $\epsilon$-truncated cone $C_{\epsilon} M$ over $M$, in the direction of $\xi$.

When $\bar{M}_{c}^{n+2}$ is a warped product $I \times_{f} F^{n+1}$, with $I \subset \mathbb{R}$, it is well known that $\xi=\left(f \circ \pi_{I}\right) \partial_{t}\left(\pi_{I}: \bar{M} \rightarrow I\right.$ being the canonical projection $)$ is a closed conformal vector field on $\bar{M}$, with conformal factor $\psi_{\xi}=f^{\prime} \circ \pi_{I}$. If we ask that $f(0)=1$, then $\Xi=\{0\} \times F$, furnished with the induced metric, is a leaf of the distribution $\{\xi\}^{\perp}$ and is isometric to $F$. Hence, one can identify an isometric immersion $\varphi: M^{n} \rightarrow F^{n+1}$ with the isometric immersion $\widetilde{\varphi}(p)=(0, \varphi(p))$, from $M^{n}$ into $\Xi=\{0\} \times F$. The flux

2010 Mathematics Subject Classification. Primary: 53C42. Secondary: 53C12.

Key words and phrases. Minimal submanifolds; Simons' formula; stability of cones.

The second author is partially supported by CNPq. The third author is partially supported by Procad-CNPq. 
of $\xi /\|\xi\|$ is given by $\Psi(t,(x, p))=(t+x, p)$, so that $\Phi(p, t)=\Psi(t, \widetilde{\varphi}(p))=(t, \varphi(p))$ and $C_{\epsilon} M$ can be identified to the immersion $\Phi: M^{n} \times[-\epsilon, 0] \rightarrow I \times_{f} F^{n+1}$, that sends $(p, t)$ to $(t, \varphi(p))$.

Our goal in this paper is to analyse the stability of $C_{\epsilon} M$ when $M^{n}$ is a closed minimal hypersurface of $F^{n+1}$. In doing so, we extend a result of Simons (cf. [10), proving the following assertion (cf. Theorem 3.3 and equations (25) and (26)).

Theorem. In the above setting, $C_{\epsilon} M$ is unstable if, and only if, $\lambda_{1}+\delta_{1}<0$, where $\lambda_{1}$ and $\delta_{1}$ are, respectively, the first eigenvalues of the linear differential operators $\mathcal{L}_{1}: C^{\infty}(M) \rightarrow C^{\infty}(M)$ and $\mathcal{L}_{2}: C_{0}^{\infty}[-\epsilon, 0] \rightarrow C^{\infty}[-\epsilon, 0]$, given by

$$
\mathcal{L}_{1}(g)=-\Delta g-\|A\|^{2} g \text { and } \quad \mathcal{L}_{2}(h)=-f^{2} h^{\prime \prime}-n f f^{\prime} h^{\prime}-c(n+1) f^{2} h,
$$

for $g \in C^{\infty}(M)$ and $h \in C_{0}^{\infty}[-\epsilon, 0]$.

Here, as in [10, $C_{0}^{\infty}[-\epsilon, 0]=\left\{h \in C^{\infty}[-\epsilon, 0] ; h(-\epsilon)=h(0)=0\right\}$.

Then, we specialize our discussion to the case of spherical cones. More precisely, we let $\mathbb{S}^{n+1}$ be the equator of $\mathbb{S}^{n+2}$ with respect to the North pole $N=(0,1)$ of $\mathbb{S}^{n+2}$, and identify each $x \in \mathbb{S}^{n+1}$ with $(x, 0) \in \mathbb{S}^{n+2}$; if we let $\bar{M}=\left(-\frac{\pi}{2}, \frac{\pi}{2}\right) \times \cos t \mathbb{S}^{n+1}$, then the mapping $(t, x) \mapsto(\cos t) x+(\sin t) N$ defines an isometry between $\bar{M}$ and $\mathbb{S}^{n+2} \backslash\{ \pm N\}$. The $\epsilon$-truncated cone $C_{\epsilon} M$ in $\mathbb{S}^{n+2}$, built over a closed minimal hypersurface $M^{n}$ of $\mathbb{S}^{n+1}$ is given by the immersion $\Phi: M^{n} \times[-\epsilon, 0] \rightarrow \mathbb{S}^{n+2}$ that maps $(x, t)$ to $(\cos t) x+(\sin t) N$. In this setting, we finish the paper by proving the following result (cf. Theorem 4.2).

Theorem. Let $M^{n}$ be a closed, oriented minimal hypersurface of $\mathbb{S}^{n+1}$. If $2 \leq n \leq$ 14 and $M^{n}$ is not totally geodesic, then $C M$ is a minimal unstable hypersurface of $\mathbb{S}^{n+2}$.

\section{On foliations Generated By CLOSED CONFORMal VECTOR FIELDS}

In what follows, $\bar{M}_{c}^{n+k+1}$ is an $(n+k+1)$-dimensional Riemaniann manifold, whose sectional curvature is constant and equal to $c$. We assume that $\bar{M}$ is furnished with a nontrivial closed conformal vector field $\xi$, i.e., $\xi \in \mathfrak{X}(M) \backslash\{0\}$ is such that $\bar{\nabla}_{X} \xi=\psi_{\xi} X$, for all $X \in \mathfrak{X}(M)$, where $\psi_{\xi}: \bar{M} \rightarrow \mathbb{R}$ is a smooth function, said to be the conformal factor of $\xi$, and $\bar{\nabla}$ denotes the Levi-Civita connection of $\bar{M}$.

From now on, the condition that $\xi \neq 0$ on $\bar{M}$ will be in force. It is immediate to check (cf. 4]) that the distribution $\left\{\xi^{\perp}\right\}$ is integrable, with leaves totally umbilical in $\bar{M}$. Let $\Xi^{n+k}$ be a leaf of such distribution, $M^{n}$ be a closed, $n$-dimensional Riemannian manifold and $\varphi: M^{n} \rightarrow \Xi^{n+k}$ be an isometric immersion. If we let $\Psi(t, \cdot)$ denote the flow of the vector field $\xi /\|\xi\|$, the compactness of $M$ assures that we can choose $\epsilon>0$ such that the map

$$
\begin{aligned}
\Phi: M^{n} \times[-\epsilon, 0] & \longrightarrow \bar{M}^{n+k+1} \\
(p, t) & \longmapsto \Psi(t, \varphi(p))
\end{aligned}
$$

is an immersion. The $\epsilon$-truncated cone over $M$, in the direction of $\xi$, which will be henceforth denoted by $C_{\epsilon} M$, is the manifold with boundary $M^{n} \times[-\epsilon, 0]$, furnished with the metric induced by $\Phi$. We observe that $C_{\epsilon} M$ is a compact, immersed submanifold of $\bar{M}_{c}^{n+k+1}$, such that $\partial\left(C_{\epsilon} M\right)=M \cup M_{\epsilon}$, where $M_{\epsilon}=$ $\{\Psi(-\epsilon, \varphi(p)) ; p \in M\}$. At times, if there is no danger of confusion, we shall refer simply to the $\epsilon$-truncated cone $C_{\epsilon} M$. 
From now on, we will frequently refer to the smooth function $\lambda: M \times[-\epsilon, 0] \rightarrow \mathbb{R}$, given by

$$
\lambda(q, t)=\exp \left(\int_{0}^{t} \frac{\psi_{\xi}}{\|\xi\|}(\Psi(s, \varphi(q))) d s\right) .
$$

The following result relates the second fundamental form of $C_{\epsilon} M$ at distinct points along the same generatrix of the cone.

Proposition 2.1. Let $A_{q}^{\eta}$ denote the shape operator of $\varphi$ at $q$, in the direction of the unit vector $\eta$, normal to $T_{q} M$ in $T_{q} \Xi$. Let $N$ denote the parallel transport of $\eta$ along the integral curve of $\xi /\|\xi\|$ that passes through $q$. If $A_{(q, t)}^{N}$ denotes the shape operator of $\Phi$ at the point $(q, t)$, in the direction of $N_{(q, t)}$, then

$$
\left\|A_{(q, t)}^{N}\right\|=\frac{1}{\lambda(q, t)}\left\|A_{q}^{\eta}\right\|
$$

Proof. Fix a point $p \in M$ and, in a neighborhood $\Omega \subset M$ of $p$, an orthonormal set $\left\{e_{1}, \ldots, e_{n}, \eta\right\}$ of vector fields, with $e_{1}, \ldots, e_{n}$ tangent to $M^{n}$ and $\eta$ normal to $M^{n}$ in $\Xi^{n+k}$. Further, ask that $A_{p}^{\eta}\left(e_{i}\right)=\lambda_{i} e_{i}(p)$, for $1 \leq i \leq n$. Let $E_{1}, \ldots, E_{n}, N$ be the vector fields on $\Phi(\Omega \times(-\epsilon, 0])$, respectively obtained from $e_{1}, \ldots, e_{n}$ and $\eta$ by parallel transport along the integral curves of $\frac{\xi}{\|\xi\|}$ that intersect $\Omega$.

If we let $\bar{R}$ denote the curvature operator of $\bar{M}$ and use the fact that $\bar{M}$ has constant sectional curvature, such a parallelism gives

$$
\begin{aligned}
\frac{d}{d t}\left\langle\bar{\nabla}_{E_{i}} N, E_{k}\right\rangle & =\left\langle\bar{\nabla}_{\frac{\xi}{|\xi|}} \bar{\nabla}_{E_{i}} N, E_{k}\right\rangle \\
& =\frac{1}{\|\xi\|}\left[\left\langle\bar{R}\left(\xi, E_{i}\right) N, E_{k}\right\rangle+\left\langle\bar{\nabla}_{E_{i}} \bar{\nabla}_{\xi} N, E_{k}\right\rangle+\left\langle\bar{\nabla}_{\left[\xi, E_{i}\right]} N, E_{k}\right\rangle\right] \\
& =\frac{1}{\|\xi\|}\left[\left\langle\bar{R}\left(\xi, E_{i}\right) N, E_{k}\right\rangle-\left\langle\bar{\nabla}_{\bar{\nabla}_{E_{i}} \xi} N, E_{k}\right\rangle\right] \\
& =-\frac{\psi_{\xi}}{\|\xi\|}\left\langle\bar{\nabla}_{E_{i}} N, E_{k}\right\rangle .
\end{aligned}
$$

Moreover, if $D$ denotes the Levi-Civita connection of $\Xi^{n+k}$, then

$$
\left\langle\bar{\nabla}_{E_{i}} N, E_{k}\right\rangle_{(p, 0)}=\left\langle D_{e_{i}} \eta, e_{k}\right\rangle_{p}=-\left\langle A^{\eta}\left(e_{i}\right), e_{k}\right\rangle_{p}=-\lambda_{i} \delta_{i k} .
$$

Equations (3) and (44) compose a Cauchy problem, whose solution is

$$
\left\langle\bar{\nabla}_{E_{i}} N, E_{i}\right\rangle_{(p, t)}=-\lambda_{i} \exp \left(-\int_{0}^{t} \frac{\psi_{\xi}}{\|\xi\|}(\varphi(p), s) d s\right)=\frac{-\lambda_{i}}{\lambda(p, t)}
$$

and, for $k \neq i$

for all $t \in(-\epsilon, 0]$.

$$
\left\langle\bar{\nabla}_{E_{i}} N, E_{k}\right\rangle_{(p, t)}=0
$$

Since $\left\langle\bar{\nabla}_{E_{i}} N, \xi\right\rangle=-\left\langle N, \bar{\nabla}_{E_{i}} \xi\right\rangle=-\psi_{\xi}\left\langle N, E_{i}\right\rangle=0$, it follows from the previous formulae that, at the point $(p, t)$,

$$
A^{N}\left(E_{i}\right)=-\left(\bar{\nabla}_{E_{i}} N\right)^{\top}=-\sum_{k=1}^{n}\left\langle\bar{\nabla}_{E_{i}} N, E_{k}\right\rangle E_{k}-\left\langle\bar{\nabla}_{E_{i}} N, \frac{\xi}{\|\xi\|}\right\rangle \frac{\xi}{\|\xi\|}=\frac{\lambda_{i}}{\lambda} E_{i},
$$

for $1 \leq i \leq n$. Finally, taking into account that $A^{N}\left(\frac{\xi}{\|\xi\|}\right)=-\left(\bar{\nabla}_{\frac{\xi}{\|\xi\|}} N\right)^{\top}=0$, we get

$$
\left\|A_{(p, t)}^{N}\right\|^{2}=\sum_{i=1}^{n}\left(\frac{\lambda_{i}}{\lambda(p, t)}\right)^{2}=\frac{1}{\lambda^{2}(p, t)}\left\|A_{p}^{\eta}\right\|^{2}
$$


Corollary 2.2. The $\epsilon$-truncated cone $C_{\epsilon} M$ is minimal in $\bar{M}$ if, and only if, $M$ is minimal in $\Xi$.

Proof. If we let $H_{(p, t)}$ be the mean curvature vector of $\Phi$ at $(p, t)$, and $H_{p}$ be that of $\varphi$ at $p$, it follows from the previous result that $\left\|H_{(p, t)}\right\|=\frac{1}{\lambda}\left\|H_{p}\right\|$. This proves the corollary.

The following technical result, which is an adapted version of Theorem 4.1 of [4], will be quite useful in the proof of Proposition 2.4. In order to state it properly, we let $\nabla$ denote the Levi-Civita connection of $C_{\epsilon} M$.

Lemma 2.3. Fix $p \in M$ and, in a neighborhood $\Omega$ of $p$ in $M$, an orthonormal frame $\left(e_{1}, \ldots, e_{n}\right)$, geodesic at $p$. If $E_{1}, \ldots, E_{n}$ are the vector fields on $\Phi(\Omega \times(-\epsilon, 0])$, respectively obtained from $e_{1}, \ldots, e_{n}$ by parallel transport along the integral curves of $\xi /\|\xi\|$ that intersect $\Omega$, then

$$
\nabla_{E_{i}} E_{i}=-\frac{\psi_{\xi}}{\|\xi\|^{2}} \xi
$$

at $(p, t)$, for all $1 \leq i \leq n$.

Proof. Choose vector fields $\left(\eta_{1}, \ldots, \eta_{k}\right)$ on $\Omega$, such that $\left(e_{1}, \ldots, e_{n}, \eta_{1}, \ldots, \eta_{k}\right)$ is an orthonormal frame adapted to the isometric immersion $\varphi$. Also, let $N_{1}, \ldots, N_{k}$ be the vector fields on $\Phi(\Omega \times(-\epsilon, 0])$, respectively obtained from $\eta_{1}, \ldots, \eta_{k}$ by parallel transport along the integral curves of $\xi /\|\xi\|$ that intersect $\Omega$. Then, the orthonormal frame $\left(E_{1}, \ldots, E_{n}, \frac{\xi}{\|\xi\|}, N_{1}, \ldots, N_{k}\right)$ on $\Phi(\Omega \times(-\epsilon, 0])$ is adapted to the isometric immersion $\Phi$.

We shall compute $\bar{\nabla}_{E_{i}} E_{i}$ at $p$ and take its tangential component along $C_{\epsilon} M$. To this end, note first of all that

$$
\left\langle\bar{\nabla}_{E_{i}} E_{i}, \xi\right\rangle=-\left\langle E_{i}, \bar{\nabla}_{E_{i}} \xi\right\rangle=-\psi_{\xi} .
$$

As before, letting $\bar{R}$ denote the curvature operator of $\bar{M}$, it follows from the parallelism of the $E_{i}$ 's, together with the fact that $\bar{M}$ has constant sectional curvature, that

$$
\begin{aligned}
\frac{d}{d t}\left\langle\bar{\nabla}_{E_{i}} E_{i}, E_{l}\right\rangle & =\frac{1}{\|\xi\|}\left\langle\bar{\nabla}_{\xi} \bar{\nabla}_{E_{i}} E_{i}, E_{l}\right\rangle \\
& =\frac{1}{\|\xi\|}\left\langle\bar{R}\left(\xi, E_{i}\right) E_{i}+\bar{\nabla}_{E_{i}} \bar{\nabla}_{\xi} E_{i}+\bar{\nabla}_{\left[\xi, E_{i}\right]} E_{i}, E_{l}\right\rangle \\
& =\frac{1}{\|\xi\|}\left(\left\langle\bar{R}\left(\xi, E_{i}\right) E_{i}, E_{l}\right\rangle-\left\langle\bar{\nabla}_{\bar{\nabla}_{E_{i}} \xi} E_{i}, E_{l}\right\rangle\right) \\
& =-\frac{\psi_{\xi}}{\|\xi\|}\left\langle\bar{\nabla}_{E_{i}} E_{i}, E_{l}\right\rangle,
\end{aligned}
$$

Also as before, let $D$ and $\nabla$ respectively denote the Levi-Civita connections of $\Xi^{n+k}$ and $M^{n}$. Since $\left(e_{1}, \ldots, e_{n}\right)$ is geodesic at $p$ (on $M$ ), we get

$$
\left\langle\bar{\nabla}_{E_{i}} E_{i}, E_{l}\right\rangle_{p}=\left\langle D_{e_{i}} e_{i}, e_{l}\right\rangle_{p}=\left\langle\nabla_{e_{i}} e_{i}, e_{l}\right\rangle_{p}=0 \text {. }
$$

Therefore, by solving Cauchy's problem formed by (17) and (8), we get

$$
\left\langle\bar{\nabla}_{E_{i}} E_{i}, E_{l}\right\rangle_{(p, t)}=0,
$$

for $-\epsilon \leq t \leq 0$.

Analogously to (7), we obtain

$$
\frac{d}{d t}\left\langle\bar{\nabla}_{E_{i}} E_{i}, N_{\beta}\right\rangle=-\frac{\psi_{\xi}}{\|\xi\|}\left\langle\bar{\nabla}_{E_{i}} E_{i}, N_{\beta}\right\rangle .
$$


On the other hand, letting $A_{\beta}: T_{p} M \rightarrow T_{p} M$ denote the shape operator of $\varphi$ in the direction of $\eta_{\beta}$ and writing $A_{\beta} e_{i}=\sum_{j=1}^{n} h_{i j}^{\beta} e_{j}$, we get

$$
\left\langle\bar{\nabla}_{E_{i}} E_{i}, N_{\beta}\right\rangle_{p}=\left\langle D_{e_{i}} e_{i}, \eta_{\beta}\right\rangle_{p}=\left\langle A_{\beta} e_{i}, e_{i}\right\rangle=h_{i i}^{\beta} .
$$

Thus, by solving Cauchy's problem formed by (10) and (11), we arrive at

$$
\left\langle\bar{\nabla}_{E_{i}} E_{i}, N_{\beta}\right\rangle_{(p, t)}=h_{i i}^{\beta} \exp \left(-\int_{0}^{t} \frac{\psi_{\xi}}{\|\xi\|}(s) d s\right)=\frac{h_{i i}^{\beta}}{\lambda(p, t)} .
$$

Finally, a simple computation shows that

$$
\left\langle\bar{\nabla}_{E_{i}} E_{i}, \frac{\xi}{\|\xi\|}\right\rangle=-\frac{\psi_{\xi}}{\|\xi\|} .
$$

Therefore, it follows from (9), (12) and (13) that, at the point $(p, t)$, we have

$$
\bar{\nabla}_{E_{i}} E_{i}=-\frac{\psi_{\xi}}{\|\xi\|} \frac{\xi}{\|\xi\|}+\frac{1}{\lambda(p, t)} \sum_{\beta=1}^{k} h_{i i}^{\beta} N_{\beta} .
$$

From this equality, (5) follows promptly.

Given a smooth function $F \in C^{\infty}\left(C_{\epsilon} M\right)$ and $t \in[-\epsilon, 0]$, we let $F_{t} \in C^{\infty}(M)$ be the (smooth) function such that $F_{t}(p)=F(p, t)$, for all $p \in M$. The next result relates the Laplacians of $F$ and $F_{t}$.

Proposition 2.4. In the above notations, for $F \in C^{\infty}\left(C_{\epsilon} M\right)$, we have

$$
\begin{aligned}
\Delta F(p, t)= & \frac{1}{\lambda^{2}(p, t)}\left(\Delta F_{t}(p)-\frac{1}{\lambda(p, t)}\left\langle\operatorname{grad}\left(F_{t}\right), \operatorname{grad}\left(\lambda_{t}\right)\right\rangle_{p}\right) \\
& +n \frac{\lambda^{\prime}(p, t)}{\lambda(p, t)} \frac{\partial F}{\partial t}+\frac{\partial^{2} F}{\partial t^{2}},
\end{aligned}
$$

where $\lambda^{\prime}$ denotes $\frac{\partial \lambda}{\partial t}$ and grad denotes gradient in $M$.

Proof. Fix a point $p \in M$ and, in a neighborhood $\Omega \subset M$ of $p$, an orthonormal frame $\left(e_{1}, \ldots, e_{n}, \eta_{1}, \ldots, \eta_{k}\right)$, adapted to $\varphi$, such that $\left(e_{1}, \ldots, e_{n}\right)$ is geodesic at $p$. As in the proof of Proposition 2.1 parallel transport this frame along the integral curves of $\xi /\|\xi\|$ to get vector fields $E_{1}, \ldots, E_{n}, N_{1}, \ldots, N_{k}$ along $\Phi(\Omega \times(-\epsilon, 0])$. Then, $\left(E_{1}, \ldots, E_{n}, \frac{\xi}{\|\xi\|}, N_{1}, \ldots, N_{k}\right)$ is an orthonormal frame adapted to the immersion $\Phi$.

The Laplacian of $F$ is given by

$$
\begin{aligned}
\Delta F & =\sum_{i=1}^{n} E_{i}\left(E_{i}(F)\right)+\frac{\xi}{\|\xi\|}\left(\frac{\xi}{\|\xi\|}(F)\right) \\
& -\sum_{i=1}^{n}\left(\nabla_{E_{i}} E_{i}\right)(F)-\left(\nabla_{\xi /\|\xi\|} \xi /\|\xi\|\right)(F) .
\end{aligned}
$$

It follows from Lemma 2.3 that

$$
\left(\nabla_{E_{i}} E_{i}\right)(F)=\left(-\frac{\psi_{\xi}}{\|\xi\|^{2}} \xi\right)(F)=-\frac{\psi_{\xi}}{\|\xi\|} \frac{\partial F}{\partial t} .
$$

Now, let us compute the summands $E_{i}\left(E_{i}(F)\right)(q, t)$, where $q \in \Omega$ and $t \in[-\epsilon, 0]$. To this end, take a smooth curve $\alpha:(-\delta, \delta) \rightarrow M$, such that $\alpha(0)=q$ and $\alpha^{\prime}(0)=e_{i}(q)$. Then, consider the parametrized surface $f:(-\delta, \delta) \times[-\epsilon, 0] \rightarrow \bar{M}$, such that

$$
f(s, t)=\Psi(t, \varphi(\alpha(s)))
$$


for $(s, t) \in(-\delta, \delta) \times[-\epsilon, 0]$. (Note that the image of $f$ is contained in $C_{\epsilon} M$.) Lemma 3.4 of [8] gives

$$
\frac{D}{d t} \frac{\partial f}{\partial s}=\frac{D}{d s} \frac{\partial f}{\partial t}=\frac{D}{d s} \frac{\xi}{\|\xi\|}=\bar{\nabla}_{\frac{\partial f}{\partial s}} \frac{\xi}{\|\xi\|}=\frac{\psi_{\xi}}{\|\xi\|} \frac{\partial f}{\partial s}+\frac{\partial f}{\partial s}\left(\frac{1}{\|\xi\|}\right) \xi
$$

which, in turn, implies

$$
\frac{d}{d t}\left\langle\frac{\partial f}{\partial s}, E_{j}\right\rangle=\frac{\psi_{\xi}}{\|\xi\|}\left\langle\frac{\partial f}{\partial s}, E_{j}\right\rangle .
$$

Since $\left\langle\frac{\partial f}{\partial s}, E_{j}\right\rangle_{(q, 0)}=\left\langle E_{i}, E_{j}\right\rangle_{(q, 0)}=\left\langle e_{i}(q), e_{j}(q)\right\rangle=\delta_{i j}$, in solving the Cauchy problem for $\left\langle\frac{\partial f}{\partial s}, E_{j}\right\rangle$ so obtained, we get

$$
\left\langle\frac{\partial f}{\partial s}, E_{i}\right\rangle_{(q, t)}=\exp \left(\int_{0}^{t} \frac{\psi_{\xi}}{\|\xi\|}(q, u) d u\right)=\lambda(q, t)
$$

and, for $j \neq i$,

$$
\left\langle\frac{\partial f}{\partial s}, E_{j}\right\rangle_{(q, t)}=0 .
$$

Moreover, direct computation shows that $\frac{d}{d t}\left\langle\frac{\partial f}{\partial s}, \xi\right\rangle=\frac{\psi_{\xi}}{\|\xi\|}\left\langle\frac{\partial f}{\partial s}, \xi\right\rangle$; but, since $\left\langle\frac{\partial f}{\partial s}, \xi\right\rangle_{(q, 0)}=\left\langle E_{i}, \xi\right\rangle_{(q, 0)}=0$, it follows from the uniqueness of the solution of a Cauchy problem that $\left\langle\frac{\partial f}{\partial s}, \xi\right\rangle_{(q, t)}=0$, for $t \in[-\epsilon, 0]$.

Since $\frac{\partial f}{\partial s}$ is tangent to the cone, the previous computations show that, at the point $(q, t)$,

$$
\frac{\partial f}{\partial s}=\sum_{j=1}^{n}\left\langle\frac{\partial f}{\partial s}, E_{j}\right\rangle E_{j}+\left\langle\frac{\partial f}{\partial s}, \frac{\xi}{\|\xi\|}\right\rangle \frac{\xi}{\|\xi\|}=\left\langle\frac{\partial f}{\partial s}, E_{i}\right\rangle E_{i}=\lambda E_{i}
$$

Therefore,

$$
E_{i}(F)(q, t)=\frac{1}{\lambda(q, t)} \frac{\partial f}{\partial s}(q, t)(F)=\frac{1}{\lambda(q, t)} d F_{t}\left(e_{i}(q)\right)=\frac{1}{\lambda(q, t)}\left\langle\operatorname{grad}\left(F_{t}\right), e_{i}\right\rangle_{q},
$$

for all points $(q, t) \in \Omega \times[-\epsilon, 0]$ and all $F \in C^{\infty}\left(C_{\epsilon} M\right)$. Thus,

$$
E_{i}\left(E_{i}(F)\right)(q, t)=\frac{1}{\lambda(q, t)}\left\langle\operatorname{grad}\left(\left(E_{i}(F)\right)_{t}\right), e_{i}\right\rangle_{q}
$$

On the other hand, at the point $q$ we get

$$
\begin{aligned}
\operatorname{grad}\left(\left(E_{i}(F)\right)_{t}\right) & =\frac{1}{\lambda_{t}} \operatorname{grad}\left\langle\operatorname{grad}\left(F_{t}\right), e_{i}\right\rangle+\left\langle\operatorname{grad}\left(F_{t}\right), e_{i}\right\rangle \operatorname{grad}\left(\frac{1}{\lambda_{t}}\right) \\
& =\frac{1}{\lambda_{t}} \operatorname{grad}\left(e_{i}\left(F_{t}\right)\right)-\left\langle\operatorname{grad}\left(F_{t}\right), e_{i}\right\rangle \frac{1}{\lambda_{t}^{2}} \operatorname{grad}\left(\lambda_{t}\right)
\end{aligned}
$$

and, hence,

$$
\begin{aligned}
E_{i}\left(E_{i}(F)\right)(q, t) & =\frac{1}{\lambda(q, t)}\left(\frac{1}{\lambda(q, t)} e_{i}\left(e_{i}\left(F_{t}\right)\right)(q)-\frac{\left\langle\operatorname{grad}\left(F_{t}\right), e_{i}\right\rangle_{q}}{\lambda^{2}(q, t)} e_{i}(q)\left(\lambda_{t}\right)\right) \\
& =\frac{1}{\lambda^{2}(q, t)}\left(e_{i}\left(e_{i}\left(F_{t}\right)\right)(q)-\frac{\left\langle\operatorname{grad}\left(F_{t}\right), e_{i}\right\rangle_{q}}{\lambda(q, t)}\left\langle\operatorname{grad}\left(\lambda_{t}\right), e_{i}\right\rangle_{q}\right),
\end{aligned}
$$

at all points $(q, t) \in \Omega \times[-\epsilon, 0]$.

By using the fact that the frame $\left(e_{1}, \ldots, e_{n}\right)$ is geodesic at the point $p$ we get, at the point $(p, t)$,

$$
\sum_{i=1}^{n} E_{i}\left(E_{i}(F)\right)=\frac{1}{\lambda^{2}}\left(\Delta F_{t}-\frac{1}{\lambda}\left\langle\operatorname{grad}\left(F_{t}\right), \operatorname{grad}\left(\lambda_{t}\right)\right\rangle\right) .
$$


If we let $(\cdot)^{\top}$ denote orthogonal projection on $T\left(C_{\epsilon} M\right)$, we compute

$$
\begin{aligned}
\nabla_{\xi /\|\xi\|} \xi /\|\xi\| & =\frac{1}{\|\xi\|} \nabla_{\xi \xi} \xi /\|\xi\|=\frac{1}{\|\xi\|}\left(\frac{1}{\|\xi\|} \nabla_{\xi} \xi+\xi\left(\frac{1}{\|\xi\|}\right) \xi\right) \\
& =\frac{1}{\|\xi\|}\left(\frac{1}{\|\xi\|}\left(\widetilde{\nabla}_{\xi} \xi\right)^{\top}-\frac{\|\xi\| \psi_{\xi}}{\|\xi\|^{2}} \xi\right) \\
& =\frac{1}{\|\xi\|}\left(\frac{1}{\|\xi\|}\left(\psi_{\xi} \xi\right)^{\top}-\frac{\psi_{\xi}}{\|\xi\|} \xi\right)=0 ;
\end{aligned}
$$

hence, $\left(\nabla_{\xi /\|\xi\|} \xi /\|\xi\|\right)(F)=0$.

Substituting this last computation in (15), and taking (16) and (18) into account, we finally arrive at

$$
\begin{aligned}
\Delta F(p, t)= & \frac{1}{\lambda^{2}(p, t)}\left(\Delta F_{t}(p)-\frac{1}{\lambda(p, t)}\left\langle\operatorname{grad}\left(F_{t}\right), \operatorname{grad}\left(\lambda_{t}\right)\right\rangle_{p}\right) \\
& +n \frac{\psi_{\xi}}{\|\xi\|} \frac{\partial F}{\partial t}(p, t)+\frac{\partial^{2} F}{\partial t^{2}}(p, t),
\end{aligned}
$$

and a simple computation shows that $\frac{\psi_{\xi}}{\|\xi\|}=\frac{\lambda^{\prime}}{\lambda}$.

\section{ON The UnStability OF Minimal CONES}

By Corollary 2.2. we know that $M^{n}$ is minimal in $\Xi^{n+k}$ if, and only if, $C_{\epsilon} M$ is minimal in $\bar{M}_{c}^{n+k+1}$. Since minimal immersions are precisely the critical points of the area functional with respect to variations that fix the boundary, for a given $M$, minimal in $\Xi^{n+k}$, it makes sense to consider the problem of stability of $C_{\epsilon} M$ with respect to normal variations that fix its boundary. In this section, we address this problem in the case in which $k=1$, i.e., when $M^{n}$ is a hypersurface of $\Xi^{n+1}$. This will extend the analysis made in [10, where $\bar{M}=R^{n+2}, \Xi=S^{n+1}$ and $\xi(x)=x$.

Throughout the rest of this paper, until further notice, we stick to the notations of the previous section. In particular, $\bar{M}$ continues to be of constant sectional curvature, equal to $c$; also, whenever we let $\eta$ denote a unit vector field normal to $M$ in $\Xi$, we shall let $N$ denote the unit vector field normal to $C_{\epsilon} M$ in $\bar{M}$, obtained by parallel transport of $\eta$ along the integral curves of $\frac{\xi}{|\xi|}$ that intersect $M$. We start with the following auxiliary result.

Lemma 3.1. Let $\Xi^{n+1}$ be oriented by the unit normal vector field $\frac{-\xi}{\|\xi\|}$, and let $M^{n}$ be a minimal hypersurface of $\Xi^{n+1}$, oriented by the unit vector field $\eta \in \mathfrak{X}(M)^{\perp} \cap$ $\mathfrak{X}(\Xi)$. If $C_{\epsilon} M$ is oriented by $N$, then its volume element is given by $\lambda^{n} d M \wedge d t$, where $d M$ stands for the volume element of $M$.

Proof. Let $\left(e_{1}, \ldots, e_{n}\right)$ be a positive orthonormal frame, defined in an open set $\Omega \subset M$. If $\left(\theta_{1}, \ldots, \theta_{n}\right)$ denotes the corresponding coframe, then $d M=\theta_{1} \wedge \ldots \wedge \theta_{n}$ in $\Omega$.

Let $E_{1}, \ldots, E_{n}$ be the vector fields on $\Phi(\Omega \times(-\epsilon, \epsilon))$ obtained from the $e_{i}$ 's by parallel transport along the integral curves of $\xi /\|\xi\|$ that intersect $\Omega$. For $p \in \Omega$, the orthonormal basis $\left(e_{1}, \ldots, e_{n}, \eta\right)$ of $T_{p} \Xi$ is positively oriented; hence, the orthonormal basis $\left(e_{1}, \ldots, e_{n}, \eta,-\frac{\xi}{\|\xi\|}\right)$ of $T_{p} \bar{M}$ is also positively oriented. It follows that the orthonormal basis $\left(E_{1}, \ldots, E_{n}, N,-\frac{\xi}{\|\xi\|}\right)_{(p, t)}$ of $T_{(p, t)} \bar{M}$ is positively oriented and, thus, $\left(E_{1}, \ldots, E_{n}, \frac{\xi}{\|\xi\|}, N\right)_{(p, t)}$ is also a positively oriented orthonormal basis of $T_{(p, t)} \bar{M}$, for all $(p, t) \in \Omega \times(-\epsilon, \epsilon)$. Therefore, $\left(E_{1}, \ldots, E_{n}, \frac{\xi}{\|\xi\|}\right)$ is a positively oriented orthonormal basis of $T_{(p, t)}\left(C_{\epsilon} M\right)$.

Now, let $\alpha_{i}:(-\delta, \delta) \rightarrow M$ be a smooth curve such that $\alpha_{i}(0)=p$ and $\alpha_{i}^{\prime}(0)=$ $e_{i}(p)$; if $f_{i}:(-\delta, \delta) \times(-\epsilon, 0] \rightarrow \bar{M}$ is the parametrized surface such that $f_{i}(s, t)=$ 
$\Psi\left(t, \varphi\left(\alpha_{i}(s)\right)\right)$, we shaw in (17) that

$$
E_{i}(p, t)=\frac{1}{\lambda(p, t)} \frac{\partial f_{i}}{\partial s}(0, t) .
$$

By the canonical identification of $T_{(p, t)}(M \times(-\epsilon, 0])$ and $T_{p} M \oplus \mathbb{R}$, we have

$$
\Phi_{*}\left(e_{i}(p) \oplus 0\right)_{(p, t)}=\left.\frac{d}{d s}\right|_{s=0} \Phi\left(\alpha_{i}(s), t\right)=\left.\frac{d}{d s}\right|_{s=0} f_{i}(s, t)=\frac{\partial f_{i}}{\partial s}(0, t)
$$

and, thus,

$$
\Phi_{*}\left(\frac{e_{i}(p)}{\lambda(p, t)} \oplus 0\right)_{(p, t)}=E_{i}(p, t) .
$$

Therefore, by using the canonical identification of $T_{\Phi(p, t)}\left(C_{\epsilon} M\right)$ and $\Phi_{*}\left(T_{(p, t)}(M \times\right.$ $(-\epsilon, 0]))$, we get

$$
\lambda^{n}(d M \wedge d t)\left(E_{1}, \ldots, E_{n}, \frac{\xi}{\|\xi\|}\right)=\lambda^{n}(d M \wedge d t)\left(\frac{e_{1}}{\lambda} \oplus 0, \ldots, \frac{e_{n}}{\lambda} \oplus 0,0 \oplus \partial t\right)=1,
$$

which concludes the proof.

Given a minimal isometric immersion $\varphi: M^{n} \rightarrow \Xi^{n+1}$, the following proposition computes the second variation of area for the corresponding $\epsilon$-truncated cone $C_{\epsilon} M$. As usual, for $F \in C^{\infty}\left(C_{\epsilon} M\right)$, we let $I(F)$ denote the index form of $C_{\epsilon} M$ in the direction of $V=F N$.

Proposition 3.2. Let $M^{n}$ be a closed, oriented, minimal hypersurface of $\Xi^{n+1}$. Suppose that the function $\lambda(p, t)$ does not depend on the point $p$, and let $N(p, t)$ denote the unit normal vector field that orients $C_{\epsilon} M$. If $F \in C^{\infty}\left(C_{\epsilon} M\right)$ is such that $F(p,-\epsilon)=F(p, 0)=0$, for each $p \in M$, then

$$
\begin{aligned}
I(F)=\int_{M \times[-\epsilon, 0]} F \lambda^{n-2} & \left(-\Delta F_{t}-n \lambda \lambda^{\prime} \frac{\partial F}{\partial t}-\lambda^{2} \frac{\partial^{2} F}{\partial t^{2}}\right. \\
& \left.-c(n+1) \lambda^{2} F-\left\|A^{\eta}\right\|^{2} F\right) d M \wedge d t .
\end{aligned}
$$

Proof. It is a classical fact (cf. 2], [10] or [11]) that

$$
I(F)=\int_{C_{\epsilon} M}\left(-F \Delta F-\left(\bar{R}+\left\|A^{N}\right\|^{2}\right) F^{2}\right) d\left(C_{\epsilon} M\right),
$$

where $\bar{R}=\overline{\operatorname{Ric}}(N, N)$, and $\overline{\operatorname{Ric}}$ denotes the Ricci tensor of $\bar{M}$. Therefore, it follows from the formulae of propositions 2.1 and 2.4 together with the fact that $\bar{M}$ has sectional curvature constant and equal to $c$ and $\lambda(p, t)$ does not depend on $p$, that the integrand of the right hand side equals

$$
\begin{aligned}
& -F\left(\frac{1}{\lambda^{2}} \Delta F_{t}+n \frac{\lambda^{\prime}}{\lambda} \frac{\partial F}{\partial t}+\frac{\partial^{2} F}{\partial t^{2}}\right)-c(n+1) F^{2}-\left\|A^{\eta}\right\|^{2} \frac{F^{2}}{\lambda^{2}}= \\
& =\frac{F}{\lambda^{2}}\left(-\Delta F_{t}-n \lambda \lambda^{\prime} \frac{\partial F}{\partial t}-\lambda^{2} \frac{\partial^{2} F}{\partial t^{2}}-c(n+1) \lambda^{2} F-\left\|A^{\eta}\right\|^{2} F\right) .
\end{aligned}
$$

Finally, it now suffices to apply the result of the previous lemma and integrate on $M \times[-\epsilon, 0]$.

Now, let $C_{0}^{\infty}[-\epsilon, 0]=\left\{g \in C^{\infty}[-\epsilon, 0] ; g(-\epsilon)=g(0)=0\right\}$. Following [10], the previous proposition motivates the introduction of the linear differential operators $\mathcal{L}_{1}: C^{\infty}(M) \rightarrow C^{\infty}(M)$ and $\mathcal{L}_{2}: C_{0}^{\infty}[-\epsilon, 0] \rightarrow C^{\infty}[-\epsilon, 0]$, given by

$$
\mathcal{L}_{1}(f)=-\Delta f-\left\|A^{\eta}\right\|^{2} f \text { and } \mathcal{L}_{2}(g)=-\lambda^{2} g^{\prime \prime}-n \lambda \lambda^{\prime} g^{\prime}-c(n+1) \lambda^{2} g .
$$

Standard elliptic theory (cf. [7]) shows that $\mathcal{L}_{1}$ can be diagonalized by a sequence $\left(f_{i}\right)_{i \geq 1}$ of smooth eigenfunctions, orthogonal in $L^{2}(M)$ and whose sequence $\left(\lambda_{i}\right)_{i \geq 1}$ 
of corresponding eigenvalues satisfy $\lambda_{1} \leq \lambda_{2} \leq \cdots \rightarrow+\infty$; moreover, each $f \in$ $C^{\infty}(M)$ can be uniquely written as $f=\sum_{i \geq 1} a_{i} f_{i}$, for some $a_{i} \in \mathbb{R}$.

On the other hand, equation $\mathcal{L}_{2}(g)=\delta g$, for $\delta \in \mathbb{R}$, is equivalent to

$$
-\lambda^{2} g^{\prime \prime}-n \lambda \lambda^{\prime} g^{\prime}-c(n+1) \lambda^{2} g-\delta g=0,
$$

or (after multiplying both sides by $-\lambda^{n-2}$ ) yet to

$$
\left(\lambda^{n} g^{\prime}\right)^{\prime}+c(n+1) \lambda^{n} g+\delta \lambda^{n-2} g=0 .
$$

Hence, the elementary theory of regular Sturm-Liouville problems (cf. 6]) shows that $\mathcal{L}_{2}$ can also be diagonalized by a sequence $\left(g_{i}\right)_{i \geq 1}$ of smooth eigenfunctions, orthogonal in $L_{w}^{2}[-\epsilon, 0]$ with respect to the weight $w=\lambda^{n-2}$ and whose sequence $\left(\delta_{i}\right)_{i>1}$ of corresponding eigenvalues satisfy $\delta_{1} \leq \delta_{2} \leq \cdots \rightarrow+\infty$; moreover, each $g \in \bar{C}_{0}^{\infty}[-\epsilon, 0]$ can be uniquely written as $g=\sum_{i>1} a_{i} g_{i}$, for some $a_{i} \in \mathbb{R}$.

In view of all of the above, the proof of the following result parallels that of Lemma 6.1.6 of [10]. For the sake of completeness, we present it here.

Theorem 3.3. With notations as in Proposition [3.2, it is possible to choose $F$ such that $I(F)<0$ if, and only if, $\lambda_{1}+\delta_{1}<0$, where $\lambda_{1}$ and $\delta_{1}$ stand, respectively, to the first eigenvalues of $\mathcal{L}_{1}$ and $\mathcal{L}_{2}$.

Proof. For a fixed $p \in M$, we have $F(p, \cdot) \in C_{0}^{\infty}[-\epsilon, 0]$. Therefore, the discussion on the diagonalization of $\mathcal{L}_{2}$ gives $F(p, t)=\sum_{j \geq 1} a_{j}(p) g_{j}(t)$, for some $a_{j} \in C^{\infty}(M)$; hence, by invoking the discussion on the diagonalization of $\mathcal{L}_{1}$, we get

$$
F(p, t)=\sum_{i, j \geq 1} a_{i j} f_{i}(p) g_{j}(t)
$$

for some $a_{i j} \in \mathbb{R}$.

It now follows from the result of Proposition 3.2 that

$$
\begin{aligned}
I(F) & =\int_{M \times[-\epsilon, 0]} \lambda^{n-2} \sum_{i, j \geq 1} a_{i j} f_{i} g_{j} \sum_{k, l \geq 1}\left(a_{k l} \mathcal{L}_{1}\left(f_{k}\right) g_{l}+a_{k l} f_{k} \mathcal{L}_{2}\left(g_{l}\right)\right) d M \wedge d t \\
& =\int_{M \times[-\epsilon, 0]} \lambda^{n-2} \sum_{i, j \geq 1} a_{i j} f_{i} g_{j} \sum_{k, l \geq 1} a_{k l}\left(\lambda_{k}+\delta_{l}\right) f_{k} g_{l} d M \wedge d t \\
& =\sum_{i, j, k, l \geq 1} a_{i j} a_{k l}\left(\lambda_{k}+\delta_{l}\right) \int_{M \times[-\epsilon, 0]} f_{i} f_{k} g_{j} g_{l} \lambda^{n-2} d M \wedge d t .
\end{aligned}
$$

From here, the orthogonality conditions on the eigenfunctions of $\mathcal{L}_{1}$ and $\mathcal{L}_{2}$ easily give

$$
I(F)=\sum_{i, j \geq 1} a_{i j}^{2}\left(\lambda_{i}+\delta_{j}\right)\left(\int_{M} f_{i}^{2} d M\right)\left(\int_{-\epsilon}^{0} g_{j}^{2} \lambda^{n-2} d t\right) .
$$

Therefore, if $I(F)<0$, then some factor $\lambda_{i}+\delta_{j}$ is negative and, hence, $\lambda_{1}+\delta_{1}<0$ (since $\lambda_{1} \leq \lambda_{i}$ and $\delta_{1} \leq \delta_{j}$ ); conversely, if $\lambda_{1}+\delta_{1}<0$, choose $F(p, t)=f_{1}(p) g_{1}(t)$ to get $I(F)<0$.

For future reference, we recall the standard variational characterization of $\lambda_{1}$ (cf. [5] or [7]): for a given $f \in C^{\infty}(M) \backslash\{0\}$, let the Rayleigh quotient of $f$ with respect to $\mathcal{L}_{1}$ be defined by

$$
R Q[f]=\frac{\int_{M}-f\left(\Delta f+\left\|A^{\eta}\right\|^{2} f\right) d M}{\int_{M} f^{2} d M}
$$

Then,

$$
\lambda_{1}=\min \left\{R Q[f] ; f \in C^{\infty}(M) \backslash\{0\}\right\},
$$

with equality if, and only if, $f$ is an eigenfunction of $\mathcal{L}_{1}$ with respect to $\lambda_{1}$. 
In what concerns $\delta_{1}$, given $g \in C_{0}^{\infty}[-\epsilon, 0] \backslash\{0\}$, let the Rayleigh quotient of $g$ with respect to (19) be defined by

$$
R Q[g]=\frac{\int_{-\epsilon}^{0} \lambda^{n}\left(\left(g^{\prime}\right)^{2}-c(n+1) g^{2}\right) d t}{\int_{-\epsilon}^{0} \lambda^{n-2} g^{2} d t}
$$

Then (cf. 6])

$$
\delta_{1}=\min \left\{R Q[g] ; g \in C_{0}^{\infty}[-\epsilon, 0] \backslash\{0\}\right\},
$$

with equality if, and only if, $g$ is an eigenfunction of $\mathcal{L}_{2}$ with respect to $\delta_{1}$.

\section{Minimal CONES IN WARPED PRODUCtS}

Let $B$ and $F$ be Riemannian manifolds and $f: B \rightarrow \mathbb{R}$ be a smooth positive function. The warped product $M=B \times{ }_{f} F$ is the product manifold $B \times F$, furnished with the Riemannian metric

$$
g=\pi_{B}^{*}\left(g_{B}\right)+\left(f \circ \pi_{B}\right)^{2} \pi_{F}^{*}\left(g_{F}\right),
$$

where $\pi_{B}$ and $\pi_{F}$ denote the canonical projections from $B \times F$ onto $B$ and $F$ and $g_{B}$ and $g_{F}$ denote the Riemannian metrics of $B$ and $F$, respectively.

In this section, we shall consider a warped product $\bar{M}_{c}^{n+2}=I \times_{f} F^{n+1}$, with $I \subset \mathbb{R}, f(0)=1$ and having constant sectional curvature, equal to $c$. By Proposition 7.42 of 9 , this last condition amounts to the fact that $F^{n+1}$ should have constant sectional curvature $k$, such that

$$
\frac{f^{\prime \prime}}{f}=-c=\frac{\left(f^{\prime}\right)^{2}-k}{f^{2}}
$$

on $I$.

In what concerns our previous discussion of cones, we get the following consequence of Proposition 2.4 when $\bar{M}=I \times{ }_{f} F$, a warped product for which $I \subset \mathbb{R}$.

Corollary 4.1. Let $\bar{M}_{c}^{n+2}=I \times_{f} F^{n+1}$, with $f(0)=1$. If $M^{n}$ is a closed Riemannian manifold and $\varphi: M^{n} \rightarrow F^{n+1}$ is an isometric immersion, then

$$
\Delta L(t, p)=\frac{1}{f^{2}(t)} \Delta L_{t}(p)+n \frac{f^{\prime}(t)}{f(t)} \frac{\partial L}{\partial t}+\frac{\partial^{2} L}{\partial t^{2}},
$$

for all $L \in C^{\infty}\left(I \times_{f} M^{n}\right)$.

Proof. It is a standard fact (cf. 9]) that, in $I \times{ }_{f} F^{n+1}$, the vector field $\xi=\left(f \circ \pi_{I}\right) \partial_{t}$ is closed and conformal, with conformal factor $\psi_{\xi}=f^{\prime} \circ \pi_{I}$. Moreover, $\xi \neq 0$, since $f$ is positive. The flux $\Psi$ of $\frac{\xi}{\|\xi\|}=\partial_{t}$ is given by

$$
\Psi\left(t,\left(t_{0}, p\right)\right)=\left(t+t_{0}, p\right),
$$

and it is clear that the submanifolds $\left\{t_{0}\right\} \times F^{n+1}$, with $t_{0} \in I$, are leaves of $\xi^{\perp}$.

Now, let $\varphi: M^{n} \rightarrow F^{n+1}$ be an isometric immersion from a closed Riemannian manifold $M^{n}$ into $F^{n+1}$. Since $f(0)=1$, the leaf $\{0\} \times F^{n+1}$ of $\xi^{\perp}$ (with the metric induced from $I \times{ }_{f} F^{n+1}$ ) is isometric to $F^{n+1}$; therefore, we can (and do) assume that $\varphi$ takes $M$ into $\{0\} \times F^{n+1}$. The compactness of $M$ guarantees the existence of $\epsilon>0$ such that the $\epsilon$-truncated cone $C_{\epsilon} M$ is given by the immersion

$$
\Phi(p, t)=\Psi(t,(0, \varphi(p)))=(t, \varphi(p)),
$$

for $t \in[-\epsilon, 0]$ and $p \in M^{n}$. (Actually, $\Phi$ continues to be an immersion even if we change $t \in[-\epsilon, 0]$ by $t \in I$.) Moreover, $C_{\epsilon} M$ is isometric to the warped product $[-\epsilon, 0] \times_{f} M^{n}$. 
In view of the above, the function $\lambda$ of (2) is such that

$$
\lambda(p, s)=\exp \left(\int_{0}^{s} \frac{\psi_{\xi}}{\|\xi\|}(\Psi(t, \varphi(p))) d t\right)=\exp \left(\int_{0}^{s} \frac{f^{\prime}}{f}(t) d t\right)=f(s) .
$$

In particular, $\lambda_{s}: M^{n} \rightarrow \mathbb{R}$ is constant, for all $s \in[-\epsilon, 0]$, and it suffices to apply the result of Proposition 2.4

From now on, let $M^{n}$ be a closed, minimal and non totally geodesic hypersurface of $F^{n+1} \approx\{0\} \times F^{n+1}$. According to the proof of the previous corollary, we shall identify the $\epsilon$-truncated cone $C_{\epsilon} M$ with the warped product $[-\epsilon, 0] \times_{f} M^{n}$, canonically immersed into $\bar{M}_{c}^{n+2}$.

If (as before) $N(t, p)$ stands for the unit normal vector field of $C_{\epsilon} M$ and $G \in$ $C^{\infty}\left(C_{\epsilon} M\right)$ is such that $G(-\epsilon, p)=G(0, p)=0$ for each $p \in M$, then Proposition 3.2 gives

$$
\begin{aligned}
I(G)=\int_{M \times[-\epsilon, 0]} G f^{n-2} & \left(-\Delta G_{t}-n f f^{\prime} \frac{\partial G}{\partial t}-f^{2} \frac{\partial^{2} G}{\partial t^{2}}\right. \\
& \left.-c(n+1) f^{2} G-\|A\|^{2} G\right) d M \wedge d t,
\end{aligned}
$$

where $\|A\|$ stands for the norm of the second fundamental form of the immersion of $M^{n}$ into $F^{n+1}$ and $\Delta$ for the Laplacian operator of $M^{n}$.

In this case, the linear differential operators $\mathcal{L}_{1}: C^{\infty}(M) \rightarrow C^{\infty}(M)$ and $\mathcal{L}_{2}$ : $C_{0}^{\infty}[-\epsilon, 0] \rightarrow C^{\infty}[-\epsilon, 0]$ are given by

$$
\mathcal{L}_{1}(g)=-\Delta g-\|A\|^{2} g
$$

for $g \in C^{\infty}(M)$, and

$$
\mathcal{L}_{2}(h)=-f^{2} h^{\prime \prime}-n f f^{\prime} h^{\prime}-c(n+1) f^{2} h,
$$

for $h \in C_{0}^{\infty}[-\epsilon, 0]$.

We want to apply Theorem 3.3 to the case in which $\bar{M}_{c}^{n+2}$ is the Euclidean sphere $\mathbb{S}^{n+2}$. To this end, let $I=\left(-\frac{\pi}{2}, \frac{\pi}{2}\right), f(t)=\cos t, F^{n+1}=\mathbb{S}^{n+1}, N=(0, \ldots, 0,1) \in$ $\mathbb{S}^{n+2}$ and consider $\mathbb{S}^{n+1}$ as the equator of $\mathbb{S}^{n+2}$ which has $N$ as North pole; also, identify $x=\left(x_{1}, \ldots, x_{n+2}\right) \in \mathbb{S}^{n+1}$ to the point $x=\left(x_{1}, \ldots, x_{n+2}, 0\right) \in \mathbb{S}^{n+2}$. With these conventions, the map

$$
(t, x) \mapsto(\cos t) x+(\sin t) N
$$

defines an isometry between $\left(-\frac{\pi}{2}, \frac{\pi}{2}\right) \times \cos t \mathbb{S}^{n+1}$ and $\mathbb{S}^{n+2} \backslash\{ \pm N\}$.

Once again, let $\varphi: M^{n} \rightarrow \mathbb{S}^{n+1}$ be a closed, minimal, non totally geodesic hypersurface of $\mathbb{S}^{n+1}$. The $\epsilon$-truncated cone $C_{\epsilon} M$ can be seen as the image of the isometric immersion

$$
\begin{aligned}
\Phi: \quad[-\epsilon, 0] \times M^{n} & \longrightarrow \mathbb{S}^{n+2} \\
(t, x) & \longmapsto(\cos t) x+(\sin t) N .
\end{aligned}
$$

In order to get an upper estimate for $\lambda_{1}$, recall from (20) and (21) that

$$
\lambda_{1} \leq \frac{\int_{M}-g\left(\Delta g+\|A\|^{2} g\right) d M}{\int_{M} g^{2} d M}
$$

for any $g \in C^{\infty}(M) \backslash\{0\}$. Following [10, let $\tau>0$ and $g_{\tau}=\left(\|A\|^{2}+\tau\right)^{1 / 2}$. Simons' formula for $\Delta\left(\|A\|^{2}\right.$ ) (cf. [3] or [10] - recall that $F$ is also of constant sectional curvature) easily gives

$$
g_{\tau} \Delta g_{\tau} \geq n\|A\|^{2}-\|A\|^{4} .
$$


Hence, by taking $g_{\tau}$ in place of $g$ in (28), we arrive at

$$
\lambda_{1} \leq-\frac{\int_{M}(n+\tau)\|A\|^{2} d M}{\int_{M}\left(\|A\|^{2}+\tau\right) d M},
$$

By letting $\tau \rightarrow 0$, and taking into account that $\int_{M}\|A\|^{2} d M>0$ (since $M$ is not totally geodesic), we get $\lambda_{1} \leq-n$.

In what concerns $\delta_{1}$, equation (26) gives

$$
\mathcal{L}_{2}(h)=-\left(\cos ^{2} t\right) h^{\prime \prime}+n(\sin t \cos t) h^{\prime}-(n+1)\left(\cos ^{2} t\right) h,
$$

so that (arguing as in the discussion that precedes the statement of Theorem 3.3) $\mathcal{L}_{2}(h)=\delta h$ is equivalent to

$$
\left(\left(\cos ^{n} t\right) h^{\prime}\right)^{\prime}+(n+1)\left(\cos ^{n} t\right) h+\delta\left(\cos ^{n-2} t\right) h=0 .
$$

It now follows from (22) and (23) that

$$
\delta_{1} \leq \frac{\int_{-\epsilon}^{0}\left(\cos ^{n} t\right)\left(\left(h^{\prime}\right)^{2}-(n+1) h^{2}\right) d t}{\int_{-\epsilon}^{0}\left(\cos ^{n-2} t\right) h^{2} d t},
$$

for every $h \in C_{0}^{\infty}[-\epsilon, 0] \backslash\{0\}$.

By taking

$$
h(t)=\frac{\sin \left(\frac{\pi}{\epsilon} t\right)}{\sqrt{\cos ^{n-2} t}}
$$

(which satisfies the boundary conditions), direct computations show that $h(t)^{2} \cos ^{n-2} t=$ $\sin ^{2}\left(\frac{\pi}{\epsilon} t\right)$,

$$
\begin{aligned}
\left(\cos ^{n} t\right) h^{\prime}(t)^{2}= & \frac{\pi^{2}}{\epsilon^{2}} \cos ^{2}\left(\frac{\pi}{\epsilon} t\right) \cos ^{2} t+\frac{(n-2)^{2}}{4} \sin ^{2}\left(\frac{\pi}{\epsilon} t\right) \sin ^{2} t \\
& +\frac{n-2}{4} \sin \left(\frac{2 \pi}{\epsilon} t\right) \sin (2 t),
\end{aligned}
$$

and

Therefore,

$$
(n+1)\left(\cos ^{n} t\right) h(t)^{2}=(n+1)\left(\cos ^{2} t\right) \sin ^{2}\left(\frac{\pi}{\epsilon} t\right)
$$

where

$$
\delta_{1} \leq \frac{I_{1}-I_{2}}{I_{3}}
$$

$$
\begin{aligned}
I_{1}= & \frac{\pi^{2}}{\epsilon^{2}} \int_{-\epsilon}^{0} \cos ^{2}\left(\frac{\pi}{\epsilon} t\right) \cos ^{2} t d t+\frac{(n-2)^{2}}{4} \int_{-\epsilon}^{0} \sin ^{2}\left(\frac{\pi}{\epsilon} t\right) \sin ^{2} t d t \\
& +\frac{n-2}{4} \int_{-\epsilon}^{0} \sin \left(\frac{2 \pi}{\epsilon} t\right) \sin (2 t) d t \\
I_{2}= & (n+1) \int_{-\epsilon}^{0}\left(\cos ^{2} t\right) \sin ^{2}\left(\frac{\pi}{\epsilon} t\right) d t \text { and } I_{3}=\int_{-\epsilon}^{0} \sin ^{2}\left(\frac{\pi}{\epsilon} t\right) d t .
\end{aligned}
$$

Finally, we observe that $\lim _{\epsilon \rightarrow \frac{\pi}{2}} I_{1}=\frac{\pi}{2}\left(1+\left(\frac{n-2}{4}\right)^{2}\right), \lim _{\epsilon \rightarrow \frac{\pi}{2}} I_{2}=(n+1) \frac{\pi}{8}$ and $\lim _{\epsilon \rightarrow \frac{\pi}{2}} I_{3}=\frac{\pi}{4}$, so that, for $\epsilon>0$ sufficiently close to $\frac{\pi}{2}$, we have

$$
\lambda_{1}+\delta_{1} \leq \frac{n^{2}}{8}-2 n+2
$$

Since this quadratic polynomial is negative for $2 \leq n \leq 14$, we have proved the following result.

Theorem 4.2. Let $M^{n}$ be a closed, oriented minimal hypersurface of $\mathbb{S}^{n+1}$. If $2 \leq$ $n \leq 14$ and $M^{n}$ is not totally geodesic, then $C M$ is a minimal unstable hypersurface of $\mathbb{S}^{n+2}$. 


\section{REFERENCES}

[1] F. J. Almgren, Jr. Some interior regularity theorems for minimal surfaces and an extension of Bernstein's theorem. Ann. of Math. 85 (1966), 277-292.

[2] J. L. M. Barbosa, M. do Carmo and J. Eschenburg. Stability of hypersurfaces of constant mean curvature in Riemannian manifolds. Math. Z. 197 (1988), 123-138.

[3] A. Caminha. On hypersurfaces into Riemannian spaces of constant sectional curvature. Kodai Math. J. 29 (2006), 185-210.

[4] A. Caminha. The geometry of closed conformal vector fields on Riemannian spaces. Bull. Braz. Math. Soc. 42 (2011), 277-300.

[5] I. Chavel. Eigenvalues in Riemannian Geometry. Academic Press, London, 1984.

[6] R. Courant and D. Hilbert. Methods of Mathematical Physics I. John Wiley \& Sons, New York, 1989.

[7] D. Gilbarg e N. Trudinger. Elliptic Partial Differential Equations of Second Order. SpringerVerlag, Berlin, 1998.

[8] M. do Carmo. Riemannian Geometry. Birkhäuser, Boston, 1992.

[9] B. O'Neill. Semi-Riemannian Geometry with Applications to Relativity, London, Academic Press (1983).

[10] J. Simons. Minimal Varieties in Riemannian Manifolds. The Annals of Mathematics (1968), 62-105.

[11] Y. Xin. Minimal Submanifolds and Related Topics. Nankai Tracts in Mathematics, World Scientific, Cingapura, 2003.

Departamento de Matemática, Universidade Federal do Piauí, Teresina, Piauí, Brazil. 64049-550

E-mail address: kelton@ufpi.edu.br

Departamento de Matemática, Universidade Federal do Ceará, Fortaleza, Ceará, BRAZIL. 60455-760

E-mail address: caminha@mat.ufc.br

Departamento de Matemática, Universidade Federal do Piauí, Teresina, Piauí, Brazil. 64049-550

E-mail address: barnabe@ufpi.edu.br 